1 Dependence of the critical Richardson number on the temperature gradient in

\title{
2 the mesosphere
}

3 Michael N. Vlasov and Michael C. Kelley

4 School of Electrical and Computer Engineering, Cornell University, Ithaca, NY 14853

5 Correspondence email: mv75@cornell.edu

6

7 Abstract

8 Maximum upper atmospheric turbulence results in the mesosphere from convective and/or

9 dynamic instabilities induced by gravity waves. For the first time, by comparing the vertical

10 accelerations induced by wind shear and the buoyancy force, it is shown that the critical

11 Richardson number $R i_{c}$ can be estimated. Dynamic instability is developed for $R i<R i_{c}$. This

12 new approach, for the first time, makes it is possible to establish and estimate the temperature

13 gradient impact on dynamic instability development. Regarding our results, $R i_{c}$ increases from

140.25 to 0.38 as the negative temperature vertical gradient increases from $\partial T / \partial z=0$ to $\partial T / \partial z \leq-9$

$15 \mathrm{~K} / \mathrm{km}$. However, $R i_{c}$ for the temperature, independent of altitude, is 0.25 , coinciding exactly with

16 the $R i_{c}$ commonly used and estimated in classical studies (Miles, 1961; Howard, 1961) and

17 subsequent papers without the temperature impact. The increase in the $R i_{c}$ value strongly

18 influences cooling, inducing the cooling rate increase. Also, our results show that criterion $R i_{c}<$

190.25 can only be used for the turbulent diffusion, which is characterized by eddies with sizes much

20 smaller than the scale height of the atmosphere. The $R i_{c}$ value increases with the increasing size

21 of the eddies, but the term "eddy diffusion" cannot be applied to transport due to the large-scale

22 eddies (Vlasov and Kelley, 2015). 
Key Words: 3334-Middle atmosphere dynamics, 3369-Thermospheric dynamics, 3379-

\section{Turbulence}

\section{Introduction}

In general, the Richardson number $R i$ can be defined as the ratio of the destruction of turbulent kinetic energy by buoyancy forces due to the production of turbulent energy by the wind shear flow. This determination leads to the relation (see, for example, Peixoto and Oort (1992))

$$
R i=\omega_{B}^{2} / S^{2}
$$

where $\omega_{B}$ is the buoyancy frequency,

$$
\omega_{B}^{2}=\frac{g}{T}\left(\frac{\partial T}{\partial z}+g / C_{p}\right),
$$

and $T$ is the temperature, $g$ is the acceleration of gravity, $C_{p}$ is the heat capacity at constant pressure, and

$$
S=\frac{\partial V}{\partial z}
$$

is the vertical shear of the horizontal wind with the velocity $V(z)$ height profile. It is generally accepted that a dynamic instability develops when the Richardson number is less than $1 / 4$, i.e., the parcel's vertical motion induced by wind shear dominates the motion induced by the buoyancy force. The former creates and the latter destroys these perturbations. Most authors use the critical Richardson number $R i_{c}<1 / 4$ without references. Some authors refer to Miles (1961) and Howard (1961). They consider the stable-stratified, horizontal shear flows of an ideal fluid. A set of studies takes into account the time-dependent shear flow and the results of laboratory experiments (Peixoto and Oort, 1992; Galperin et al., 2007). However, we could not find papers on the critical Richardson number that take the mesospheric conditions into account. Miles and other authors (Abarbanel et al., 1984; Ligniéres et al., 1999; Galperin et al., 2007) did not consider the 
temperature's influence on the $R i_{c}$ value. However, the eddy turbulence peak is observed in the mesosphere or the lower thermosphere where the large negative and positive gradients of the temperature occur. We could find just one paper [Hysell et al., 2012] on the estimate of the $R i_{c}$ value in the lower thermosphere. Using the data on observations of the sporadic $E$ layer, Hysell et al. (2012) inferred the parameters of wind shear corresponding to the irregularities observed in the layer and estimated the $R i_{c}$ value of 0.75 . However, the authors used the wrong formula for the background density, resulting in densities much larger than the observed atmospheric density corresponding to the hydrostatic equilibrium. It is shown in Appendix 3 how $0.7<R i_{c}<0.8$ can be found due to the background density used by Hysell et al. (2012). overriding its inertial effects is the Richardson number given by formula (1) in Miles (1961), which can be written as

$$
R i=-g \frac{\partial \rho}{\partial z} /\left\{\rho\left[\frac{\partial V}{\partial z}\right]^{2}\right\}
$$

where $\rho$ is the density and $V$ is the horizontal wind velocity. This formula can be rewritten as

$$
\left(\frac{\partial V}{\partial z}\right)^{2}=-\frac{g}{R i} \frac{1}{\rho} \frac{\partial \rho}{\partial z}
$$

62 This initial formula will be used here to estimate the accelerations induced by wind shear and the 63 buoyancy forces under mesospheric conditions.

64 The goal of this paper is to estimate the critical Richardson number, $R i_{c}$, corresponding to the equilibrium between the buoyancy force and the force induced by wind shear in the mesosphere. 


\section{Acceleration Induced by Wind Shear}

We start from formula (5) corresponding to the initial equation used by Miles (1961) (here, formula (4)). Miles considers an uncompressible fluid but the adiabatic expansion/compression should be taken into account in the upper atmosphere. Differentiating the adiabatic relation $p T^{-\gamma /(\gamma-1)}=$ const corresponding to Poisson's equation where $p=\rho \kappa T / m$ and $p$ is the pressure; $m$ is the mean molecular mass; $\gamma=C_{p} / C_{v} ; C_{p}$ and $C_{v}$ are the heat capacities at constant pressure and volume; $\gamma /(\gamma-1)=1+N / 2 ; N=5$ is the number of degrees of freedom for diatomic gas; and $\kappa$ is the Boltzmann's constant, it is possible to get the adiabatic expansion equation

$$
\frac{1}{\rho} \frac{\partial \rho}{\partial z}=\frac{N}{2} \frac{1}{T} \frac{\partial T}{\partial z}
$$

(see the derivation of this formula in Appendix1), and according to formula (5):

$$
\left(\frac{\partial V}{\partial z}\right)^{2}=-\frac{g}{R i} \frac{N}{2 T} \frac{\partial T}{\partial z}
$$

Taking into account $\operatorname{Ri}(\partial V / \partial z)^{2}=\omega_{B}^{2}=(g / T)\left(\partial T / \partial z+g / C_{p}\right)$ and using formula (6), the temperature gradient in the parcel with upward motion and adiabatic expansion can be given by the equation

$$
\frac{\partial T}{\partial z}=-\frac{g}{(1+N / 2) C_{p}}
$$

and

$$
T=T_{0}-\frac{g}{\left(1+\frac{N}{2}\right) C_{p}}\left(z-z_{0}\right)
$$

By substituting formulas (8) and (9) in formula (7) multiplied by $\left(z-z_{0}\right)$, it is possible to obtain the formula

$$
a_{w s}=\frac{g^{2} N\left(z-z_{0}\right)}{2 R i\left[T_{0} C_{p}(1+N / 2)-g\left(z-z_{0}\right)\right]}
$$


Ann. Geophys. Discuss., https://doi.org/10.5194/angeo-2018-71

Manuscript under review for journal Ann. Geophys.

Discussion started: 17 July 2018

(c) Author(s) 2018. CC BY 4.0 License.

where

$$
a_{w s}=\left(\frac{\partial V}{\partial z}\right)^{2}\left(z-z_{0}\right)
$$

92 is the acceleration in wind shear. As can be seen from Fig. 1, this acceleration increases with the increase of the vertical size of the wind shear layer. Note that this size cannot exceed $1-2 \mathrm{~km}$

94 according to the experimental data (Larsen, 2002). The $a_{w s}$ dependence on the altitude is linear because $g\left(z-z_{0}\right) \ll T_{0} C_{p}(1+N / 2)$ for $-z_{0}<2 \mathrm{~km}$.

96

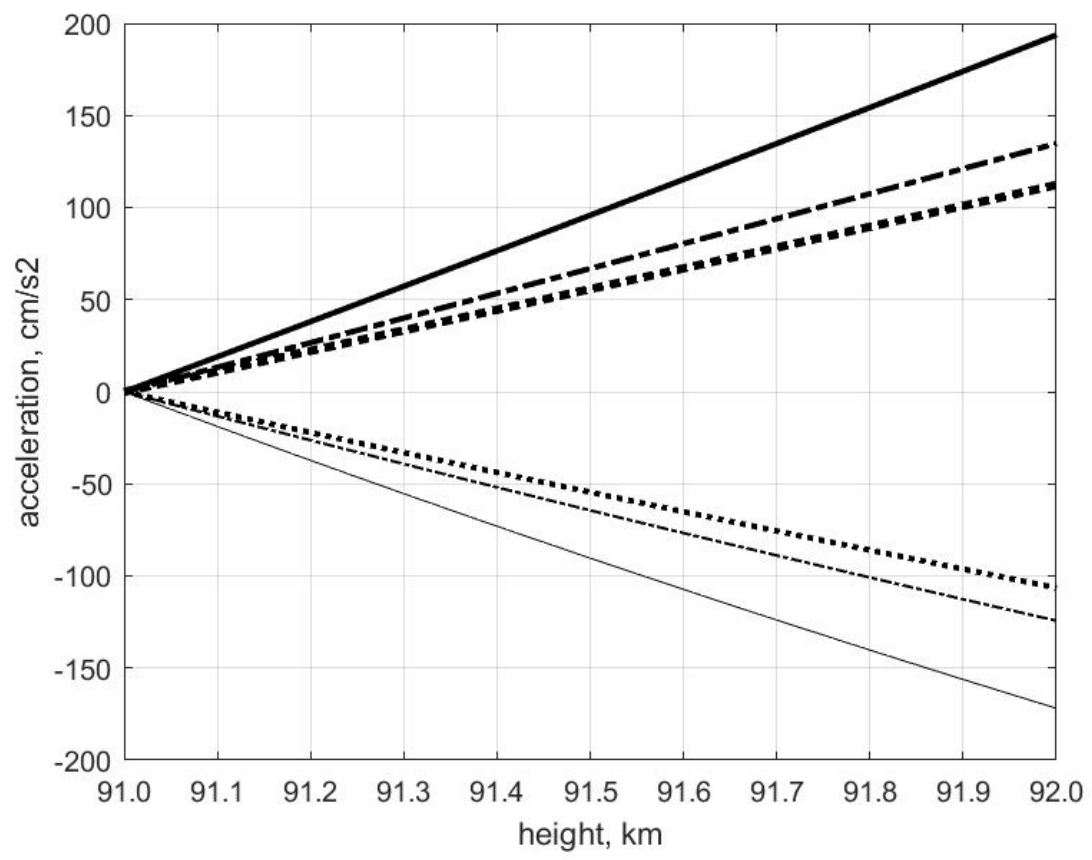

Figure 1. The height profiles of the wind shear $a_{w s}>0$ and buoyant $a_{B}<0$ accelerations calculated by formulas (11) and (15), respectively, with $T_{0}=140 \mathrm{~K}$ and $R i_{c}=0.25$ (solid curves), with $T_{0}=$ $140 \mathrm{~K}$ and $R i_{c}=R / C_{p}=0.286$ (dashed-dotted curves), and with $T_{0}=200 \mathrm{~K}$ and $R i_{c}=0.286$ (dotted curves). 


\section{Acceleration Induced by the Buoyancy Force}

The buoyancy force is $F_{B}=g\left(\rho_{A}-\rho_{D}\right)$ where $\rho_{A}$ and $\rho_{D}$ are the background atmospheric density and the disturbed density, respectively. The acceleration is given by

$$
a_{B}=g\left[\left(\rho_{A}-\rho_{D}\right) \rho_{D}\right] .
$$

The atmospheric density distribution can be given by

$$
\rho_{A}=\rho_{A 0} \exp \left[-\left(z-z_{0}\right) / H_{A}\right]
$$

for $d T_{A} / d z=0$ in the mesopause and the formula

$$
\rho_{A}=\rho_{A 0}\left\{\left[T_{A 0}-G\left(z-z_{0}\right)\right] / T_{A 0}\right\}^{(m g / \kappa G-1)}
$$

111 for $d T_{A} / d z=G<0$ below the mesopause, and $H_{A}=\kappa T_{A 0} / \mathrm{mg}$ is the scale height of the 112 atmospheric gas. By integrating equation (6) with the temperature and temperature gradient given 113 by formulas (8) and (9), it is possible to get the disturbed density distribution $\left(T_{0}=T_{A 0}\right)$,

$$
\rho_{D}=\rho_{A 0}\left[\frac{T_{0}-\frac{G\left(z-z_{0}\right)}{C_{p}(1+N / 2)}}{T_{0}}\right]^{N / 2},
$$

and the acceleration corresponding to the buoyancy force can be written as

$$
a_{B}=g\left[\left(\frac{\rho_{A}}{\rho_{D}}\right)-1\right]=g \frac{\rho_{A 0} e^{-\frac{\left(z-z_{0}\right)}{H_{A}}}}{\rho_{A 0}\left[\frac{T_{0}-\frac{g\left(z-z_{0}\right)}{C_{p}\left(1+\frac{N}{2}\right)}}{T_{0}}\right]^{\frac{N}{2}}}-g
$$

117 for $d T_{A} / d z=0$. As seen from Fig. 1, there is very good agreement between the $a_{w s}$ and $a_{B}$

118 absolute values for $R i_{c}=0.25$, and $T_{0}=140 \mathrm{~K}$ and $T_{0}=200 \mathrm{~K}$ for the vertical size of a stable wind

119 shear layer that is less than $400 \mathrm{~m}$. The $a_{w s}$ value becomes larger than the $a_{B}$ value for $z-z_{0}>$

$120400 \mathrm{~m}$, which means that the $R i_{c}$ value should be increased. The turbulence develops if $\alpha_{w s}$ is

121 larger than the $\alpha_{B}$ that corresponds to $R i<R i_{c}$. We emphasize that the perturbation scale sizes 
Ann. Geophys. Discuss., https://doi.org/10.5194/angeo-2018-71

Manuscript under review for journal Ann. Geophys.

Discussion started: 17 July 2018

(c) Author(s) 2018. CC BY 4.0 License.

induced by wind shear do not exceed 1-2 km, according to the observations (see Lübken (1997)).

Note that formula (13b) should be used instead of formula (13a) in the nominator of formula (15)

124 for atmospheric temperature distribution with $\frac{d T_{A}}{d z}<0$. As can be seen from Fig. 2, the $a_{B}$ values

125 significantly decrease in this case, since the atmospheric density given by formula (13b) is larger

126 and the density gradient is less than the density and gradient corresponding to formula (13a). The

127 small buoyancy force corresponds to the small density gradient. This dependence explains the $a_{B}$

128 reduction with the $T_{A}$ decrease.

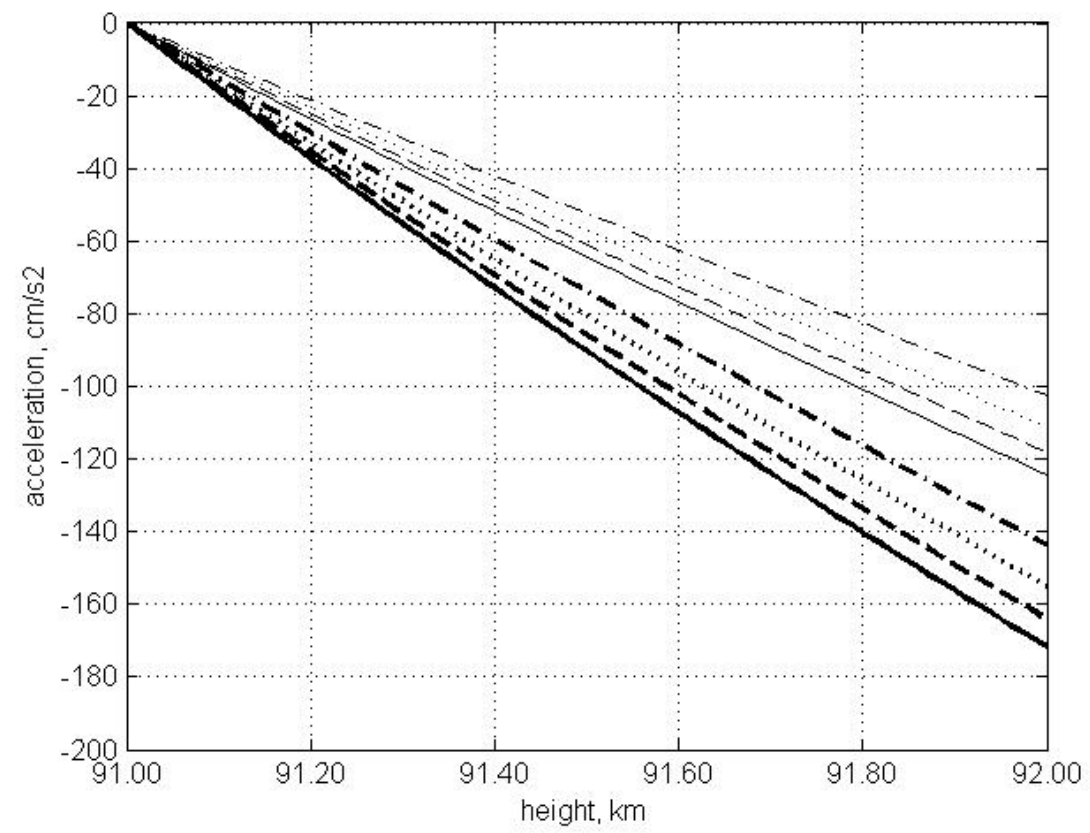

Figure 2. The height profiles of the acceleration of the buoyancy force calculated by formula (15)

132 with the nominator $\rho_{A 0}\left\{\left[T_{A 0}-G\left(z-z_{0}\right)\right] / T_{A 0}\right\}^{(m g / \kappa G-1)}$ for $T_{0}=T_{A 0}=140 \mathrm{~K}$ and $200 \mathrm{~K}$ (thick

133 and thin curves, respectively) and $G=1,2.8$, and $5 \mathrm{~K} / \mathrm{km}$ (dashed, dotted and dashed-dotted curves,

134 respectively), and calculated by formula (15) (solid curves). 


\section{Estimating the Richardson Number}

Using formulas (11) and (15) in the equation $a_{w s}+a_{B}=0$, the formula for $R i_{c}$ can be inferred:

The $R i_{c}$ values calculated by formula (16) and this formula with $\left\{\left[T_{0}-G\left(z-z_{0}\right)\right] / T_{0}\right\}^{(m g / \kappa G-1)}$

140 (see formula (13b) instead of the exponential term) are shown in Figs. 3a and 3b. The $R i_{c}$ values

141 increase with increasing altitude, corresponding to the vertical expansion of the region of the stable

wind shear. However, according to the experimental data (Larsen, 2002; Kelley et al., 2003;

Bishop et al., 2004), the wind shears are very unstable. As mentioned above, the size scales of the density perturbations do not exceed $1-2 \mathrm{~km}$, according to the observations. A more accurate consideration of eddy turbulence (Vlasov and Kelley, 2015) concludes that the scale size of density perturbations $l$ should be much less than the scale height of atmospheric gas, $l<<H_{A}$ and $l<<4$ $\mathrm{km}$ for $T_{A}=T_{0}=140 \mathrm{~K}$ and $l<<5.7 \mathrm{~km}$ for $T_{A}=T_{0}=200 \mathrm{~K}$. However, this restriction can only apply to turbulence corresponding to the eddy diffusion approximation (Vlasov and Kelley, 2015). As seen from Fig. 3a, the $R i_{c}$ value of 0.25 corresponds to perturbations with scales less than 10 $\mathrm{m}$, and the $R i_{c}$ values reach 0.256 and 0.263 for $l=200 \mathrm{~m}$ and $400 \mathrm{~m}$ and for $T_{A 0}=140 \mathrm{~K}$ and 0.254 and 0.257 for $T_{0}=200 \mathrm{~K}$, respectively. The $R i_{c}$ value of 0.25 corresponds to the mean value $l=27.3 \mathrm{~m}$ obtained by Lübkin (1997), using the measured spectrum of the density fluctuation. Vlasov and Kelley (2015) reconsidered the results of Kelley et al. (2003) and found that the spectrum scale fluctuations inferred from the meteor train turbulence observations can be approximated by Heisenberg's formula with $l=119 \mathrm{~m}$, and eddies with very large scales may 
Ann. Geophys. Discuss., https://doi.org/10.5194/angeo-2018-71

Annales

Manuscript under review for journal Ann. Geophys.

Discussion started: 17 July 2018

(c) Author(s) 2018. CC BY 4.0 License.

Discussions

(c) (1)

156 occur in the narrow layer of localized turbulence. As can be seen from Fig. $3 \mathrm{~b}$, the $R i_{c}$ values

157 increase with the increase in the negative gradient of the temperature and can reach almost 0.36 .

158

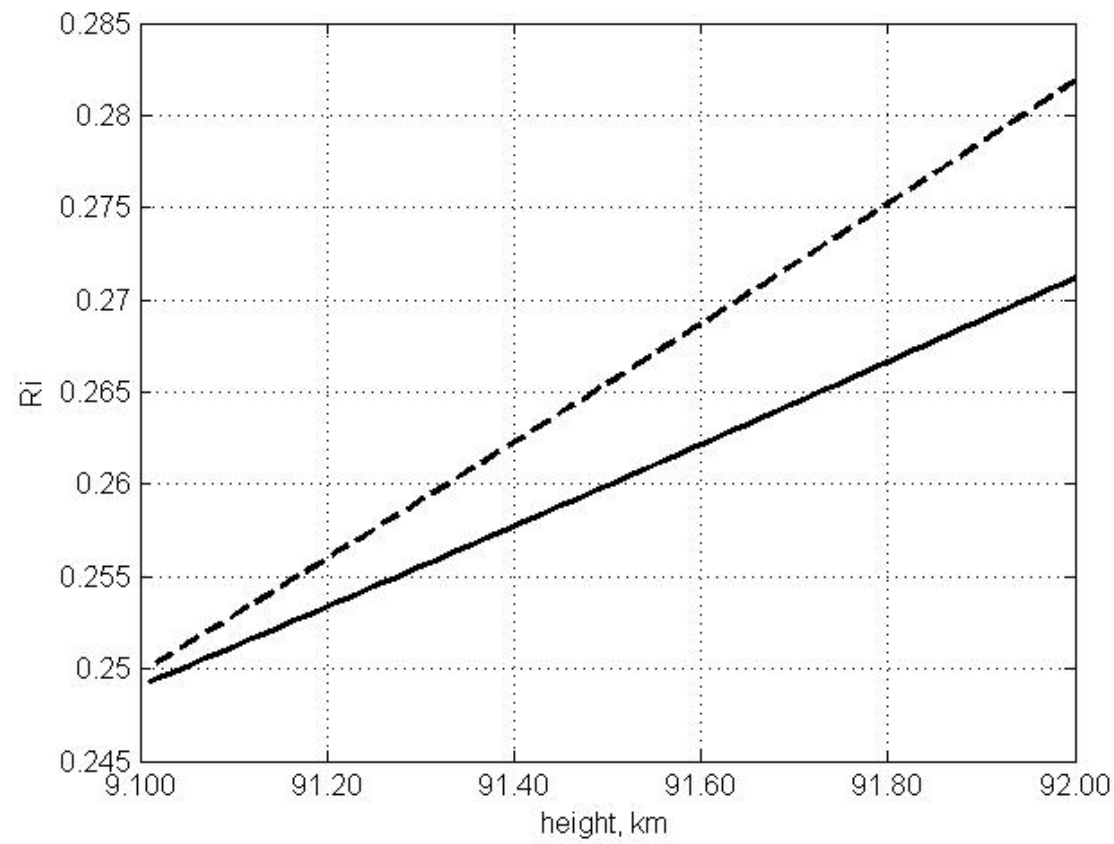

159

160 Figure 3a. The height profiles of the critical Richardson number calculated by formula (16) with

$161 T_{0}=140 \mathrm{~K}$ and $200 \mathrm{~K}$ (dashed and solid lines, respectively).

162 
Ann. Geophys. Discuss., https://doi.org/10.5194/angeo-2018-71

Manuscript under review for journal Ann. Geophys.

Discussion started: 17 July 2018

(c) Author(s) 2018. CC BY 4.0 License.

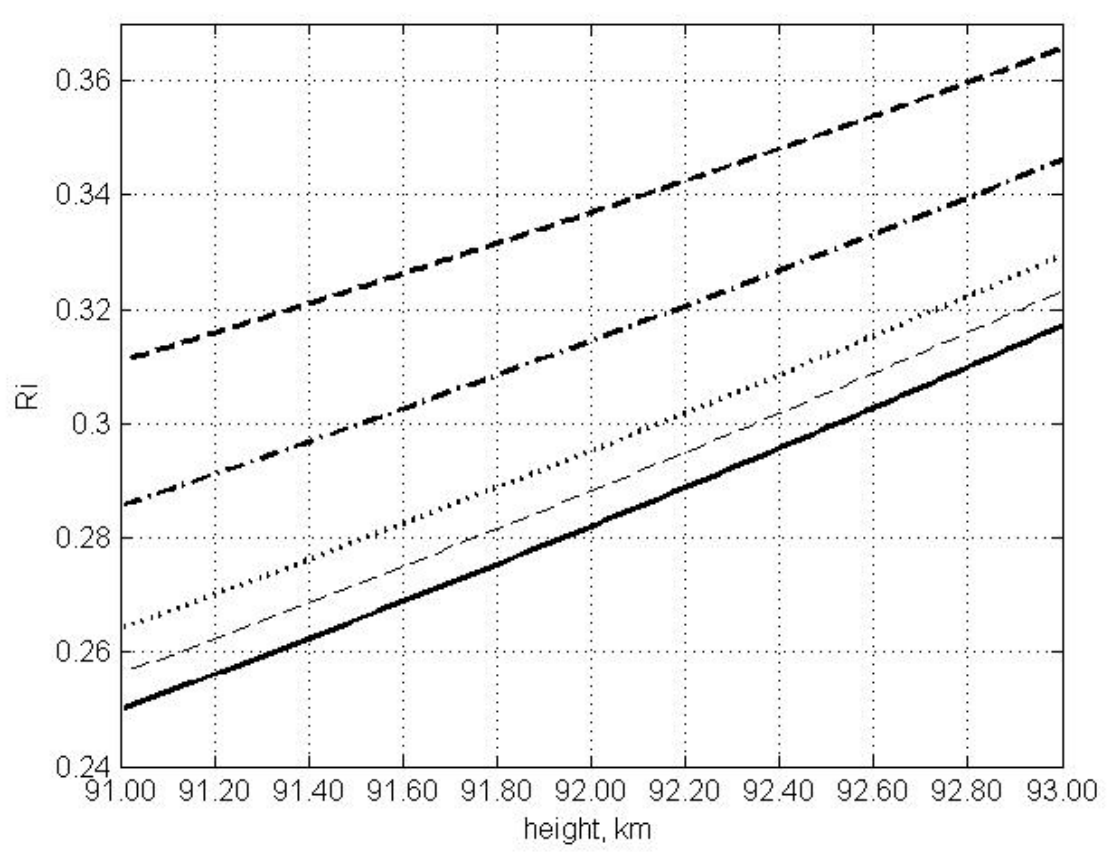

164 Figure 3b. The height profiles of the critical Richardson number calculated by formula (16) with

$165\left\{\left[T_{0}-G\left(z-z_{0}\right)\right] / T_{0}\right\}^{(m g / \kappa G-1)}$ instead of the exponential term for the $T_{0}=140 \mathrm{~K}$ with $d T / d z=$

$166 G<0$ with $|G|=0.2,1,3$, and $5 \mathrm{~K} / \mathrm{km}$ (dashed thin, dotted, dashed-dotted and dashed thick curves,

167 respectively) and calculated by formula (16) (solid thick curve).

168

169 Thus, turbulence can develop with $R i_{c}>0.25$ for wind shears with a vertical size of $1-2 \mathrm{~km}$,

170 but this turbulence may not correspond to eddy diffusion. The scales of the density fluctuations

171 are very small (for example, see Lübken (1997)) that correspond to $z \rightarrow z 0$. However, the $R i_{c}$ value

172 estimation for $z \rightarrow z_{0}$ is problematic because, in this case, the numerator and denominator in

173 formula (16) try to attain zero. This uncertainty can be solved using L'Hospital's rule, leading to

174 the formula (see Appendix 2) 
Ann. Geophys. Discuss., https://doi.org/10.5194/angeo-2018-71

Manuscript under review for journal Ann. Geophys.

Discussion started: 17 July 2018

(c) Author(s) 2018. CC BY 4.0 License.

$$
R i_{c}=\frac{0.5 g N}{g(1+N / 2)^{2}-0.5 g N-G C_{p}(1+N / 2)}
$$

176 for the $R i_{c}$ limit value for $z \rightarrow z_{0}$. This formula corresponds to the limit value formula (16) with

177 the term $\left\{\left[T_{0}-G\left(z-z_{0}\right)\right] / T_{0}\right\}^{(m g / k G-1)}$ instead of the term $\exp \left[-\left(z-z_{0}\right) / H_{A}\right]$. The $R i_{c}$

178 dependence on the negative temperature gradient, given by formula (17), is shown in Fig. 4. The

$179 G$ increase improves the conditions for the dynamic instability development. Note that the $R i_{c}$

180 value for $G=0$ coincides with the results of Miles (1961) and the commonly used value of $R i_{c}$.

181

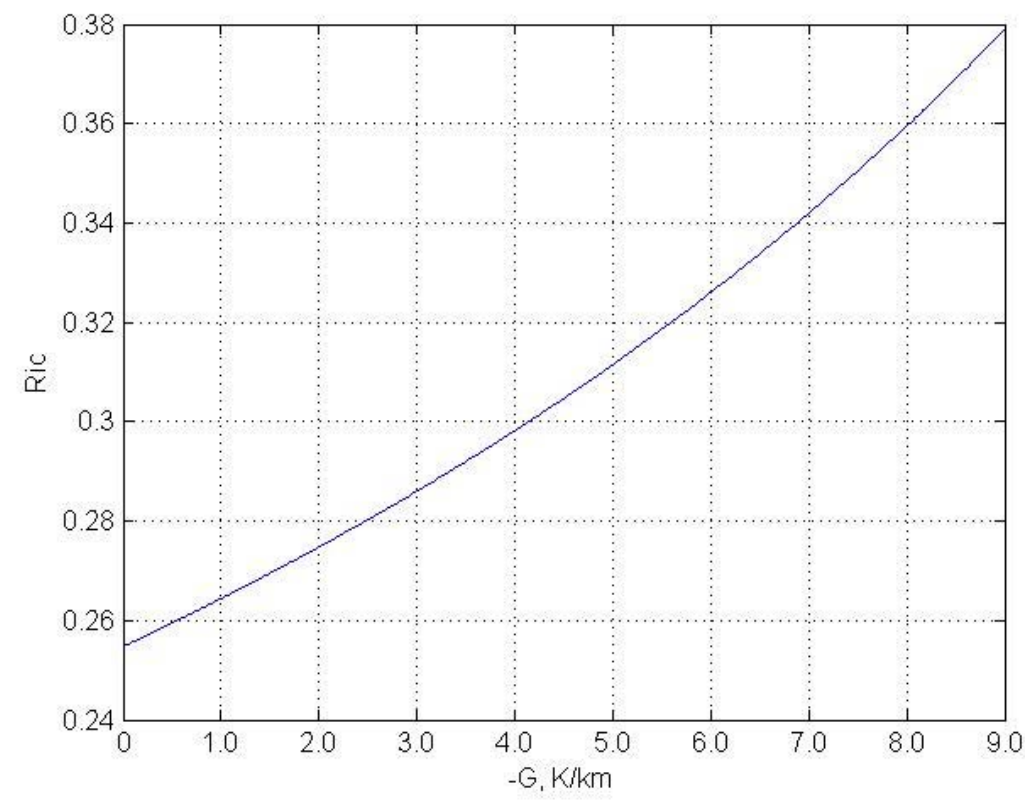

Figure 4. The dependence of the Richardson number $R i_{c}$ on the temperature negative gradient calculated by formula (17).

\section{The Influence of $R i_{c}$ Dependence on $G$ on Cooling in the Mesosphere}




$$
Q_{e d}=\frac{\partial}{\partial z}\left[K_{e h} C_{p} \rho\left(\frac{\partial T}{\partial z}+\frac{g}{C_{p}}\right)\right]+K_{e h} \rho \frac{g}{T b}\left(\frac{\partial T}{\partial z}+\frac{g}{C_{p}}\right)
$$

where $K_{e h}$ is the coefficient of the eddy heat transport, $\rho$ is the undisturbed gas density, and $b$ is a

dimensionless constant given by the relation obtained using the results of Gordiets et al. (1982),

$$
b=R i_{c} /\left(P-R i_{c}\right)
$$

where $P$ is the turbulent Prandtl number. According to equation (18), the $Q_{e d}$ value is given in units $\operatorname{erg} \times \mathrm{Cm}^{-3} \times S^{-1}$. The $K_{e h}$ value is given by

$$
K_{e h}=b \varepsilon / \omega_{B}^{2},
$$

where $\varepsilon$ is the energy dissipation rate, and $b$ can be given by formula (19). The vertical distribution

of the $\varepsilon$ value in the turbulent layer can be approximated by the Gaussian function

$$
\varepsilon=\varepsilon_{m} \exp \left[-\left(z-z_{m}\right)^{2} / h^{2}\right],
$$

where $h$ is half of the layer thickness and $\varepsilon_{m}$ is the $\varepsilon$ value at the altitude of the layer peak $z_{m}$.

Using this approximation, dividing equation (18) by $\rho C_{p}$ and substituting formula (20) with $b=$

$R i /(P-R i)$ and $T=T_{0}+G\left(z-z_{0}\right)$, equation (18) can be written in units $K / s$ as

$$
Q_{e d}=\varepsilon_{m} \exp \left[-\frac{\left(z-z_{m}\right)^{2}}{h^{2}}\right]\left\{\frac{\left[T_{0}+G\left(z-z_{0}\right)\right]}{g\left(\frac{P}{R i_{c}}-1\right)}\left[-\frac{2\left(z-z_{m}\right)}{h^{2}}-\frac{\frac{m g}{\kappa}}{T_{0}+G\left(z-z_{0}\right)}\right]+\frac{1}{C_{p}}\right\} .
$$

Using the $R i_{c}$ dependence on the temperature gradient given by formula (17), the impact of the Richardson number on the cooling rates can be estimated. According to the results in Fig. 5, the cooling rates increase by a factor of 2.2 for $0.25<R i_{c}<0.38$ corresponding to $0 \leq G \leq-9 \mathrm{~K} / \mathrm{km}$, but the $G$ value influence on the cooling for $R i_{c}=$ const $=0.25$ is very small (curves near the thick solid curve). Note that the turbulence induced by the large wind shear may not correspond to the 
Ann. Geophys. Discuss., https://doi.org/10.5194/angeo-2018-71

Manuscript under review for journal Ann. Geophys.

Discussion started: 17 July 2018

(c) Author(s) 2018. CC BY 4.0 License.

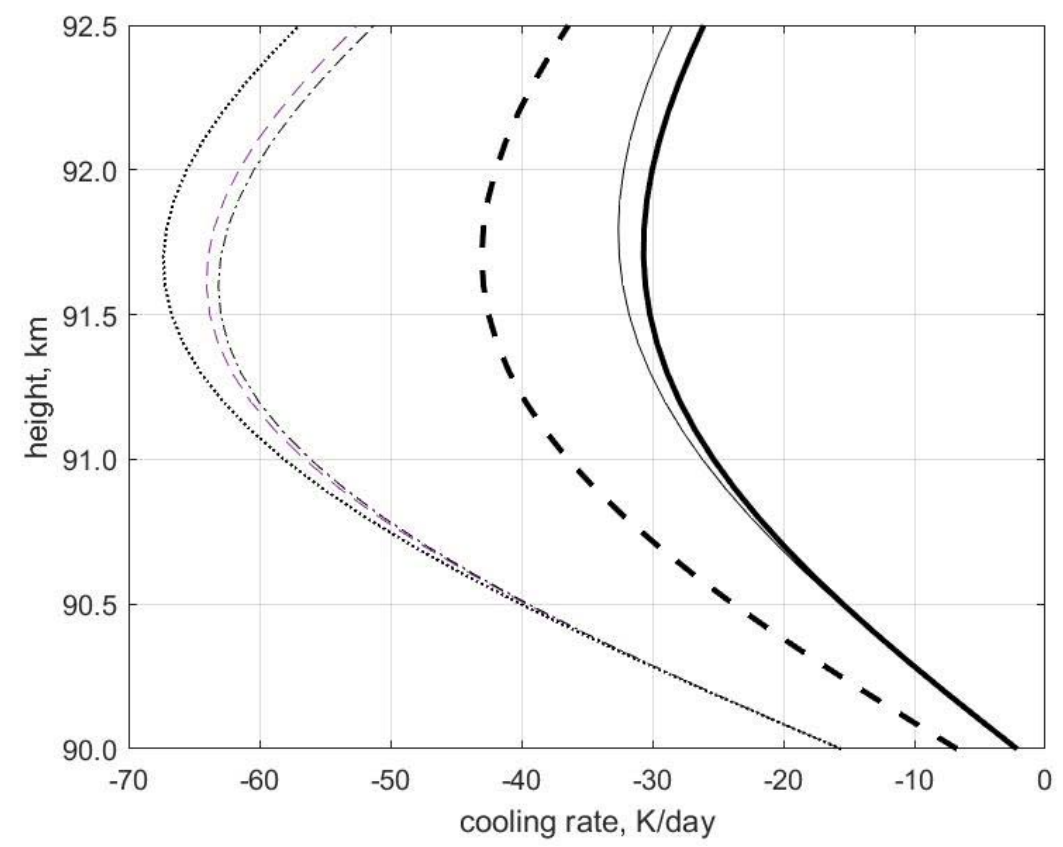

212 Figure 5. The cooling rates calculated by equation (22) with $G=0 \mathrm{~K} / \mathrm{km}-R i=0.25, G=-3 \mathrm{~K} / \mathrm{km}$

$-R i=0.286, G=-5 \mathrm{~K} / \mathrm{km}-R i=0.31, \mathrm{G}=-7 \mathrm{~K} / \mathrm{km}-R i=0.34, G=-8 \mathrm{~K} / \mathrm{km}-R i=0.36, \mathrm{G}=-9$

$\mathrm{K} / \mathrm{km}-R i=0.38$ (thick solid, dashed and dashed-dotted curves and thin dotted, solid curves and thick dotted curve, respectively) and the $Q_{e d}$ values calculated with $R i=0.25$ and the $G$ values

216 from $-3 \mathrm{~K} / \mathrm{km}$ to $-9 \mathrm{~K} / \mathrm{km}$ are shown by curves near the thick solid curve.

\section{Conclusions}

219 For the first time, by comparing the accelerations in wind shear and the buoyancy force, it is 
be estimated and the dynamic instability developed for $R i<R i_{c}$. This new approach is very different from the approach used in classical studies (Miles, 1961) and subsequent papers. Note that Miles and the other authors did not consider the temperature's influence on dynamic instability development. However, the mesosphere is characterized by the negative temperature gradient, and the turbulence peak is observed in this region. For the first time, it has been estimated and established that the $R i_{c}$ value depends on the temperature gradient. The $R i_{c}$ value increases with the negative mesospheric temperature gradient increase. It should be emphasized that our estimated $R i_{c}$ value is exactly the same as the $R i_{c}$ value of 0.25 estimated by Miles (1961) and other authors and does not depend on the temperature for $d T / d z=0$.

The Richardson number dependence on the temperature gradient influences the cooling rates induced by eddy turbulence. These rates significantly increase with an increasing $R i_{c}$, but the influence of the negative temperature gradient on the cooling for $R i_{c}=$ const $=0.25$ is very small.

Also, our results show that criterion $R i_{c}=0.25$ can be used for turbulent diffusion that is characterized by eddies with a size that is much less than the scale height of the atmosphere. The $R i_{c}$ value increases with the increase in the vertical size of the wind shear (see Fig. 3a), but there is a problem with applying the term "eddy diffusion" to momentum and heat transport because of the large-scale eddies in this case (Vlasov and Kelley, 2015).

239 In general, our results show that the criterion $R i_{c}=0.25$ can only be applied to turbulence with small scales corresponding to the eddy diffusion. This diffusion provides the mixing of neutral constituents and their diffusive separation as a result of the competition between eddy and

242 molecular diffusion. In this case, the criterion $R i_{c}=0.25$ is necessary and sufficient, but not for 243 the more complicated shears mentioned above and observed in the lower thermosphere. 
Ann. Geophys. Discuss., https://doi.org/10.5194/angeo-2018-71

Annales

Manuscript under review for journal Ann. Geophys.

Discussion started: 17 July 2018

(c) Author(s) 2018. CC BY 4.0 License.

Discussions

(c) (i)

244

245

246

247

248

250

251

252

253

254

255

256

257

258

259

260

261

262

263

\section{Appendix 1}

Derivation of formula (6) in the paper. We start by using the adiabatic equation $p T^{-\gamma /(\gamma-1)}=$ const:

$$
\frac{\partial}{\partial z}\left[p T^{-\gamma /(\gamma-1)}\right]=0
$$

$$
\frac{\partial}{\partial z}\left[R \rho T \times T^{-1-N / 2}\right]=R\left[\frac{\partial \rho}{\partial z} T^{-N / 2}-\rho \frac{N}{2} T^{-1-N / 2} \frac{\partial T}{\partial z}\right]=0 .
$$

Dividing this equation by $\rho$ and multiplying by $T^{-N / 2}$, it is possible to get the adiabatic expansion equation

$$
\frac{1}{\rho} \frac{\partial \rho}{\partial z}=\frac{N}{2} \frac{1}{T} \frac{\partial T}{\partial z}
$$

\section{Appendix 2}

Derivation of formula (17) for $\partial T / \partial z=G=0$ :

$$
R i_{c}=\frac{\left[1-\frac{g\left(z-z_{0}\right)}{B}\right]^{N / 2}}{\left[1-\frac{g\left(z-z_{0}\right)}{B}\right]^{N / 2}-\exp \left[-\frac{\left(z-z_{0}\right)}{H_{A}}\right]} \frac{0.5 g N\left(z-z_{0}\right)}{B-g\left(z-z_{0}\right)}=\frac{F(z)}{\varphi(z)}
$$

where $B=T_{0} C_{p}(1+N / 2)$ and

$$
\frac{\partial F}{\partial z}=-\frac{N g}{2 B}\left[1-\frac{g\left(z-z_{0}\right)}{B}\right]^{N / 2-1} \frac{0.5 g N\left(z-z_{0}\right)}{B-g\left(z-z_{0}\right)}+\left[1-\frac{g\left(z-z_{0}\right)}{B}\right]^{N / 2} \frac{0.5 g N\left[B-g\left(z-z_{-} 0\right)\right]+0.5 g N\left(z-z_{0}\right) g}{\left[B-g\left(z-z_{0}\right)\right]^{2}} .
$$

For $z=z_{0}$,

$$
\frac{\partial F}{\partial z}=\frac{0.5 \mathrm{gNB}}{B^{2}}=\frac{0.5 \mathrm{gN}}{B}
$$


Ann. Geophys. Discuss., https://doi.org/10.5194/angeo-2018-71

$$
\frac{\partial \emptyset}{\partial z}=-\frac{N_{g}}{2 B}\left[1-\frac{g\left(z-z_{0}\right)}{B}\right]^{N / 2-1}+\frac{1}{H_{A}} \exp \left[-\frac{\left(z-z_{0}\right)}{H_{A}}\right] .
$$

265 For $z=z_{0}$,

266

$$
\frac{\partial \emptyset}{\partial z}=-\frac{N g}{2 B}+\frac{1}{H_{A}} .
$$

267 Finally, we have a very simple formula:

268

$$
R i=\frac{0.5 g N}{B \frac{m g}{k T_{0}}-0.5 g N}=\frac{0.5 N}{\left(1+\frac{N}{2}\right)^{2}-0.5 N}=0.256 \text { for } N=5, G=0
$$

269

and for $G<0$,

$$
\frac{\partial \emptyset}{\partial z}=-\frac{0.5 N_{g}}{B}-\frac{\partial}{\partial z}\left\{\frac{\left[T_{0}-G\left(z-z_{0}\right)\right]}{T_{0}}\right\}^{\frac{m g}{\kappa G}-1}=-\frac{0.5 N_{g}}{B}-\left(\frac{m g}{\kappa G}-1\right)\left(\frac{-G}{T_{0}}\right) \text { for } z=z_{0}
$$

$$
\frac{\left(\frac{\partial F}{\partial z}\right)}{\left(\frac{\partial \varnothing}{\partial z}\right)}=\frac{0.5 g N}{B\left[-\frac{0.5 g N}{B}+\frac{m g}{\kappa T_{0}}-\frac{G}{T_{0}}\right]}=-\frac{0.5 g N}{-0.5 g N+g\left(1+\frac{N}{2}\right)^{2}-\frac{G B}{T_{0}}}=\frac{0.5 g N}{\left(1+\frac{N}{2}\right)^{2} g-0.5 N_{g}-G C_{p}(1+N / 2)}
$$

\section{Appendix 3}

The equation used by Hysell et al. $(2009,2012)$ is

$$
N^{2}=-\frac{g}{\rho_{0}} \frac{\partial \rho_{0}}{\partial z}=\frac{g}{T}\left(\frac{\partial T}{\partial z}+\frac{g}{C_{p}}\right)
$$

Here, $N^{2}$ is the buoyancy frequency square and $\rho_{0}$ is the background density. This equation is incorrect because first, the buoyancy frequency for incompressible fluid is not equal to the frequency for compressible fluid, and second, the background density given by the equation

$$
\frac{1}{\rho_{0}} \frac{\partial \rho_{0}}{\partial z}=-\frac{1}{T}\left(\frac{\partial T}{\partial z}+\frac{g}{C_{p}}\right)
$$

is much larger than the density given by the equation

$$
\frac{1}{\rho_{A}} \frac{\partial \rho_{A}}{\partial z}=-\frac{1}{T}\left(\frac{\partial T}{\partial z}+\frac{g}{R}\right)
$$


for hydrostatic equilibrium corresponding to real atmospheric conditions. For example, the scale height of the density is $H=\kappa T(1+N / 2) / m g$ corresponding to equation (A2) where $\partial T / \partial z=0$ is larger by a factor of 3.5 than the scale height of the background atmospheric density $H=$ $\kappa T / m g$ corresponding to equation (A3). The atmospheric density inferred from equation (A2) with $\partial T / \partial z=G$ is given by the formula

$$
\rho_{A}=\rho_{A 0}\left\{\left[T_{A 0}+G\left(z-z_{0}\right)\right] / T_{A 0}\right\}^{(-m g / \kappa G(1+0.5 N)-1)} .
$$

This formula is similar to formula (13b) but with $G>0$ and $-m g / \kappa G(1+0.5 N)$ instead of $-m g / \kappa G$. The density given by formula (A4) is much larger than the density given by formula (13b) for $G>0$. Substituting formula (A4) instead of the exponential term in equation (16) and using L'Hospital's rule, it is possible to get the equation

$$
R i_{c}=\frac{0.5 g N}{g(1+0.5 N)-0.5 g N+G C_{p}(1+0.5 N)}=\frac{0.5 g N}{g+G C_{p}(1+0.5 N)}
$$

instead of equation (17).

According to Fig. 2 in Hysell et al. (2012), a sporadic $E$ layer with significant irregularities was observed by Arecibo INR at a height of around $110 \mathrm{~km}$ at 19:30 - 20:30 LT on July 2, 2010 in the lower thermosphere. The authors used the data on this layer to infer the parameters of the wind shear and then, using a numerical model, they estimated the $R i_{c}$ value of 0.75 for the dynamic instability corresponding to the observed irregularities in this region. According to the data shown in Fig. 2 (Hysell et al., 2012), the temperature gradient in the instability at around $110 \mathrm{~km}$ is $G=$ $6-8 \mathrm{~K} / \mathrm{km}$ and the $R i_{c}$ value can be found to be $0.8-0.65$, respectively, according to equation (A5). It follows that the large $R i_{c}$ value of 0.75 estimated by the numerical model of Hysell et al. (2012) can only result from the large density used instead of the correct background density. In this case, the $R i_{c}$ value does not depend on the specific features of wind shear inferred by the authors and used in the numerical model. According to equation (17) with $G>0$ and the 
Ann. Geophys. Discuss., https://doi.org/10.5194/angeo-2018-71

Manuscript under review for journal Ann. Geophys.

Discussion started: 17 July 2018

(c) Author(s) 2018. CC BY 4.0 License. with $G$ increasing from 0 to $8 \mathrm{~K} / \mathrm{km}$.

307

\section{Competing Interests}

309 The authors declare that they have no conflict of interest.

310

\section{Acknowledgments}

312 Work at Cornell University was funded by the School of Electrical and Computer Engineering

313 and the Cornell Podell Emeriti Awards for Research and Scholarship (PEARS) Program through

314 CAPE. This paper is entirely theoretical and no data have been used.

\section{References}

317 Abarbanel, H., Holm, D., Marsden, J., and Ratiu, T.: Richardson number criterion for the nonlinear

318 stability of three-dimensional stratified flow, Phys. Rev. Lett., 52, 2352-2355, 1984.

319 Bishop, R. L., Larsen, M. F., Hecht, J. H., Liu, A. Z., and Gardner, C. S.: TOMEX: Mesospheric

320 and lower thermospheric diffusivity and instability layers, J. Geophys. Res., 109, D02S03, 321 doi:10.1029/2002JD003079, 2004.

322 Galperin, B., Sukoriansky, S., and Anderson, P. S.: On the critical Richardson number in stably 323 stratified turbulence, Atmos. Sci. Lett., 8(3), 65-69, 2007.

324 Gordiets, B. F., Kulikov, Yu. N., Markov, M. N., and Marov, M. Ya: Numerical modelling of the 325 thermospheric heat budget, J. Geophys. Res., 87, 4504-4514, 1982.

326 Howard, L.: Note on a paper of John W. Miles, J. Fluid Mech., 10, 509-512, 1961. 
328 Dynamic instability in the lower thermosphere inferred from irregular sporadic E layers, J.

329 Geophys. Res., 117, A08305, doi:10.1029/2012JA017910, 2012.

330 Kelley, M. C., Kruschwitz, C. A., Gardner, C. S., Drummond, J.D., and Kane, T. J.: Mesospheric

331 turbulence measurements from persistent Leonid meteor train observations, J. Geophys. Res., 332 108, 8454, doi:10.1029/2002JD002392, 2003.

333 Larsen, M. F.: Winds and shears in the mesosphere and lower thermosphere: Results from four 334 decades of chemical release wind measurements, J. Geophys. Res., 107, 1215, 335 doi:1029/2001JA000218, 2002.

336 Ligniéres, F., Califano, F., and Mangeney, A.: Shear layer instability in a highly diffusive stably 337 stratified atmosphere, Astron. and Astrophys., 349, 1027-1036, 1999.

338 Lübken, F. J.: Seasonal variation of turbulent energy dissipation rates at high latitudes as 339 determined by in situ measurements of neutral density fluctuations, J. Geophys. Res., 102, $340 \quad 13,441-13,456,1997$.

341 Miles, J.: On the stability of heterogeneous shear flows, J. Fluid Mech., 10, 496-508, 1961.

342 Peixoto, J. P., and Oort, A. H.: Physics of Climate. New York: Springer-Verlag, 1992.

343 Vlasov, M. N., and Kelley, M. C.: Estimates of eddy turbulence consistent with seasonal variations 344 of atomic oxygen and its possible role in the seasonal cycle of mesopause temperature, Ann. 345 Geophys., 28, 2103-2110, 2010.

346 Vlasov, M. N., and Kelley, M. C.: Eddy diffusion coefficients and their upper limits based on 347 application of the similarity theory, Ann. Geophys., 33, 857-864, 2015. 